\title{
GROTHENDIECK'S INEQUALITY IN THE NONCOMMUTATIVE SCHWARTZ SPACE
}

\author{
RUPERT H. LEVENE AND KRZYSZTOF PISZCZEK
}

\begin{abstract}
In the spirit of Grothendieck's famous inequality from the theory of Banach spaces, we study a sequence of inequalities for the noncommutative Schwartz space, a Fréchet algebra of smooth operators. These hold in non-optimal form by a simple nuclearity argument. We obtain optimal versions and reformulate the inequalities in several different ways.
\end{abstract}

\section{INTRODUCTION}

The noncommutative Schwartz space $\mathcal{S}$ is a weakly amenable mconvex Fréchet algebra whose properties have been investigated in several recent papers, see e.g. $[2,3,13,14]$. It is not difficult to see that as a Fréchet space, $\mathcal{S}$ is nuclear. From this, we can easily deduce the following analogue of Grothendieck's inequality, which we call Grothendieck's inequality in $\mathcal{S}$ : there exists a constant $K>0$ so that for any continuous bilinear form $u: \mathcal{S} \times \mathcal{S} \rightarrow \mathbb{C}$ and any $n \in \mathbb{N}$, there exists $k \in \mathbb{N}$ such that for every $m \in \mathbb{N}$ and any $x_{1}, \ldots, x_{m}, y_{1}, \ldots, y_{m} \in \mathcal{S}$, we have

$$
\left|\sum_{j=1}^{m} u\left(x_{j}, y_{j}\right)\right| \leqslant K\|u\|_{n}^{*}\left\|\left(x_{j}\right)\right\|_{k}^{\mathrm{RC}}\left\|\left(y_{j}\right)\right\|_{k}^{\mathrm{RC}}
$$

The norms on the right hand side arise naturally from the definition of $\mathcal{S}$, as explained in Section 2 below. Our goal in this note is to show that in fact $k=2 n+1$ always suffices, and that this is best possible.

This appears to be the first result concerning Grothendieck's inequality in the category of Fréchet algebras; to the best of our knowledge,

2010 Mathematics Subject Classification. Primary: 47A30, 47A07, 47L10. Secondary: 47A63.

Key words and phrases. noncommutative Grothendieck inequality, noncommutative Schwartz space, m-convex Fréchet algebra, bilinear form, state.

The research of the second-named author has been supported by the National Center of Science, Poland, grant no. UMO-2013/10/A/ST1/00091.

The authors are grateful to the anonymous referee, whose comments greatly improved the presentation of this paper. 
all previous results along these lines concern Banach spaces (including $\mathrm{C}^{*}$-algebras, general Banach algebras and operator spaces). For Fréchet algebras, Grothendieck's inequality seems to have a specific flavour. Every Fréchet space (and a fortiori, every Fréchet algebra) which appears naturally in analysis is nuclear, meaning that all tensor product topologies are equal. Since Grothendieck's inequality can be understood as the equivalence of two tensor products, it seems that we can take inequality (1) for granted. The interesting question that remains is then optimality.

This paper is divided into four sections. In the remainder of this section, we recall a $\mathrm{C}^{*}$-algebraic version of Grothendieck's inequality due to Haagerup, and then review the definition and the basic properties of $\mathcal{S}$ which we require. In Section 2 we explain how nuclearity gives Grothendieck's inequality in $\mathcal{S}$, and we estimate the constants $K$ and $k$. Section 3 then settles the optimality question for $k$ via a matricial construction. We conclude with a short section containing several reformulations of the inequality.

1.1. Grothendieck's inequality. Pisier's survey article [12] is a comprehensive reference for Grothendieck's inequality. This presents many equivalent formulations and applications of this famous result, and recounts its evolution from 'commutative' [5] to 'noncommutative'. Of these reformulations and extensions, Haagerup's noncommutative version most closely resembles (1), and we state it here for the convenience of the reader.

Theorem 1 ([6], [12, Theorem 7.1]). Let $A$ and $B$ be $C^{*}$-algebras. For any bounded bilinear form $u: A \times B \rightarrow \mathbb{C}$ and any finite sequence $\left(x_{j}, y_{j}\right)$ in $A \times B$, we have

$$
\left|\sum u\left(x_{j}, y_{j}\right)\right| \leqslant 2\|u\|\left\|\left(x_{j}\right)\right\|^{\mathrm{RC}}\left\|\left(y_{j}\right)\right\|^{\mathrm{RC}}
$$

where $\left\|\left(x_{j}\right)\right\|^{\mathrm{RC}}:=\max \left\{\left\|\sum x_{j}^{*} x_{j}\right\|^{\frac{1}{2}},\left\|\sum x_{j} x_{j}^{*}\right\|^{\frac{1}{2}}\right\}$.

\subsection{The noncommutative Schwartz space. Let}

$$
s=\left\{\xi=\left(\xi_{j}\right)_{j \in \mathbb{N}} \in \mathbb{C}^{\mathbb{N}}:|\xi|_{n}:=\left(\sum_{j=1}^{+\infty}\left|\xi_{j}\right|^{2} j^{2 n}\right)^{\frac{1}{2}}<+\infty \text { for all } n \in \mathbb{N}\right\}
$$

denote the so-called space of rapidly decreasing sequences. This space becomes Fréchet when endowed with the sequence $\left(|\cdot|_{n}\right)_{n \in \mathbb{N}}$ of norms defined above. The basis $\left(U_{n}\right)_{n \in \mathbb{N}}$ of zero neighbourhoods of $s$ is defined by $U_{n}:=\left\{\xi \in s:|\xi|_{n} \leqslant 1\right\}$. The topological dual $s^{\prime}$ of $s$ is the so-called 
space of slowly increasing sequences, namely

$$
\left\{\eta=\left(\eta_{j}\right)_{j \in \mathbb{N}} \in \mathbb{C}^{\mathbb{N}}:|\eta|_{n}^{\prime}:=\left(\sum_{j=1}^{+\infty}\left|\eta_{j}\right|^{2} j^{-2 n}\right)^{\frac{1}{2}}<+\infty \text { for some } n \in \mathbb{N}\right\}
$$

where the duality pairing is given by $\langle\xi, \eta\rangle:=\sum_{j \in \mathbb{N}} \xi_{j} \overline{\eta_{j}}$ for $\xi \in s$, $\eta \in s^{\prime}$.

The noncommutative Schwartz space $\mathcal{S}$ is the Fréchet space $L\left(s^{\prime}, s\right)$ of all continuous linear operators from $s^{\prime}$ into $s$, endowed with the topology of uniform convergence on bounded sets. The formal identity map $\iota: s \hookrightarrow s^{\prime}$ is a continuous embedding and defines a product on $\mathcal{S}$ by $x y:=x \circ \iota \circ y$ for $x, y \in \mathcal{S}$. There is also a natural involution on $\mathcal{S}$ given by $\left\langle x^{*} \xi, \eta\right\rangle:=\langle\xi, x \eta\rangle$ for $x \in \mathcal{S}, \xi, \eta \in s^{\prime}$. With these operations, $\mathcal{S}$ becomes an $\mathrm{m}$-convex Fréchet $*$-algebra. The inclusion map $\mathcal{S} \hookrightarrow \mathcal{K}\left(\ell_{2}\right)$ is continuous, and in fact it is a spectrum-preserving $*$-homomorphism [3]. Moreover [13, Proposition 3], an element $x \in \mathcal{S}$ is positive (i.e., $x=y^{*} y$ for some $y \in \mathcal{S}$ ), if and only if the spectrum of $x$ is contained in $[0,+\infty)$, or equivalently $\langle x \xi, \xi\rangle \geq 0$ for all $\xi \in s^{\prime}$. On the other hand, by [3, Cor. 2.4] and [4, Theorems 8.2, 8.3], the topology of $\mathcal{S}$ cannot be given by a sequence of $\mathrm{C}^{*}$-norms. This causes some technical inconvenience (e.g. there is no bounded approximate identity in $\mathcal{S}$ ) meaning we cannot apply $\mathrm{C}^{*}$-algebraic techniques directly.

\section{The INEQUALity}

Let $\left(\|\cdot\|_{n}\right)_{n \in \mathbb{N}}$ be a non-decreasing sequence of norms which gives the topology of $\mathcal{S}$. For $u: \mathcal{S} \times \mathcal{S} \rightarrow \mathbb{C}$ a continuous bilinear form, we write

$$
\|u\|_{n}^{*}:=\sup \left\{|u(x, y)|: x, y \in \mathcal{U}_{n}\right\}
$$

where $\mathcal{U}_{n}=\left\{x \in \mathcal{S}:\|x\|_{n} \leqslant 1\right\}$; similarly, for a functional $\phi \in \mathcal{S}^{\prime}$, we write

$$
\|\phi\|_{n}^{*}:=\sup \left\{|\phi(x)|: x \in \mathcal{U}_{n}\right\} .
$$

Following Pisier [11, p. 316], for $k \in \mathbb{N}$ and $x_{1}, x_{2}, \ldots, x_{m} \in \mathcal{S}$, we write

$$
\left\|\left(x_{j}\right)\right\|_{k}^{\mathrm{RC}}=\max \left\{\left\|\sum_{j=1}^{m} x_{j}^{*} x_{j}\right\|_{k}^{\frac{1}{2}},\left\|\sum_{j=1}^{m} x_{j} x_{j}^{*}\right\|_{k}^{\frac{1}{2}}\right\} .
$$

Relative to our choice of norms $\|\cdot\|_{n}$, we have now defined each term in our hoped-for inequality (1). We will now reformulate it using tensor products.

For $\mathrm{C}^{*}$-algebras, such a reformulation is standard. Indeed, by [6, Theorem 1.1] (formulated along the lines of [8, Theorem 2.1]), Haagerup's noncommutative Grothendieck inequality entails the existence of a $K>$ 
0 such that for any $\mathrm{C}^{*}$-algebras $A, B$ and $z$ in the algebraic tensor product $A \otimes B$, we have $\|z\|_{\pi} \leqslant K\|z\|_{a h}$ where $\|\cdot\|_{\pi}$ is the projective tensor norm and $\|\cdot\|_{a h}$ is the absolute Haagerup tensor norm [8, p. 164] on $A \otimes B$, given by

$$
\|z\|_{a h}=\inf \left\|\sum_{j=1}^{m}\left|x_{j}\right|^{2}\right\|^{\frac{1}{2}}\left\|\sum_{j=1}^{m}\left|y_{j}\right|^{2}\right\|^{\frac{1}{2}} .
$$

Here $|x|=\left(\frac{1}{2}\left(x^{*} x+x x^{*}\right)\right)^{\frac{1}{2}}$ for $x$ an element of a $\mathrm{C}^{*}$-algebra, and the infimum is taken over all representations $z=\sum_{j=1}^{m} x_{j} \otimes y_{j}$ where $\left(x_{j}, y_{j}\right) \in A \times B$.

We proceed similarly for $\mathcal{S}$. For $x \in \mathcal{S}$, let $|x|^{2}=\frac{1}{2}\left(x^{*} x+x x^{*}\right) \in$ $\mathcal{S}$ and consider the sequence of absolute Haagerup tensor norms (\|. $\left.\|_{a h, n}\right)_{n \in \mathbb{N}}$ on the algebraic tensor product $\mathcal{S} \otimes \mathcal{S}$ given by

$$
\|z\|_{a h, n}:=\inf \left\|\sum_{j=1}^{m}\left|x_{j}\right|^{2}\right\|_{n}^{\frac{1}{2}}\left\|\sum_{j=1}^{m}\left|y_{j}\right|^{2}\right\|_{n}^{\frac{1}{2}}
$$

where the infimum runs over all ways to represent $z=\sum_{j=1}^{m} x_{j} \otimes y_{j}$ in $\mathcal{S} \otimes \mathcal{S}$. As usual, we write $\left(\|\cdot\|_{\pi, n}\right)_{n \in \mathbb{N}}$ for the sequence of projective tensor norms on $\mathcal{S} \otimes \mathcal{S}$.

Just as in the $\mathrm{C}^{*}$-algebra case, inequality (1) will follow once we show that the sequences of projective and absolute Haagerup tensor norms are equivalent on $\mathcal{S} \otimes \mathcal{S}$. In fact, the equivalence of these norms follows immediately from the nuclearity of $\mathcal{S}$ (see [9, Theorem 28.15] and [7, Ch. 21, $\S 2$, Theorem 1] for details). On the other hand, the optimal values of $k$ and $K$ (depending on $n$ and our choice of norms $\left(\|\cdot\|_{n}\right)_{n \in \mathbb{N}}$ ) for which (1) hold are not given by such general considerations. These optimal parameters will be denoted by $\kappa(n):=k_{\text {best }}$ and $K_{n}:=K_{\text {best }}$.

Henceforth, we focus only on the sequence of norms $\left(\|\cdot\|_{n}\right)_{n \in \mathbb{N}}$ where

$$
\|x\|_{n}:=\sup \left\{|x \xi|_{n}: \xi \in U_{n}^{\circ}\right\}, \quad n \in \mathbb{N}, x \in \mathcal{S}
$$

and $U_{n}^{\circ}=\left\{\xi \in s^{\prime}:|\xi|_{n}^{\prime} \leqslant 1\right\}$. In other words, $\|x\|_{n}$ is the norm of $x \in \mathcal{S}$, considered as a Hilbert space operator from $H_{n}^{\prime}:=\ell_{2}\left(\left(j^{-n}\right)_{j}\right)$ to $H_{n}:=\ell_{2}\left(\left(j^{n}\right)_{j}\right)$. This sequence does indeed induce the topology of $\mathcal{S}$. In this context, we will estimate $K_{n}$ and compute the exact values of $\kappa(n)$.

We start with the following result, which can be compared with [13, Lemma 8]. To fix some useful notation, for $n \in \mathbb{N}$ we define an infinite diagonal matrix $d_{n}:=\operatorname{diag}\left(1^{n}, 2^{n}, 3^{n}, 4^{n}, \ldots\right)$ which we consider as an isometry $d_{n}: \ell_{2} \rightarrow H_{n}^{\prime}$ and simultaneously as an isometry $d_{n}: H_{n} \rightarrow \ell_{2}$. 
Proposition 2. Let $n \in \mathbb{N}$. We have

(i) $\|x\|_{n}=\sup \left\{\langle x \xi, \xi\rangle: \xi \in U_{n}^{\circ}\right\}$ for every positive $x \in \mathcal{S}$;

(ii) $\|x\|_{n}^{2} \leqslant\left\|x^{2}\right\|_{2 n}$ for every self-adjoint $x \in \mathcal{S}$; and

(iii) $\|x\|_{n}^{2} \leqslant\left\|x^{*} x\right\|_{2 n}^{\frac{1}{2}}\left\|x x^{*}\right\|_{2 n}^{\frac{1}{2}}$ for every $x \in \mathcal{S}$.

Moreover, inequalities (ii) and (iii) are sharp.

Proof. (i) Observe that $\|x\|_{n}=\left\|d_{n} x d_{n}\right\|_{\mathcal{B}\left(\ell_{2}\right)}$. Furthermore, since $x$ is positive, $d_{n} x d_{n}$ is positive and we have

$$
\begin{aligned}
\|x\|_{n} & =\left\|d_{n} x d_{n}\right\|_{\mathcal{B}\left(\ell_{2}\right)}=\sup \left\{\left\langle x d_{n} \xi, d_{n} \xi\right\rangle:|\xi|_{\ell_{2}} \leqslant 1\right\} \\
& =\sup \left\{\langle x \xi, \xi\rangle:|\xi|_{n}^{\prime} \leqslant 1\right\} .
\end{aligned}
$$

(ii) For $x$ self-adjoint, we have

$$
\left\|x^{2}\right\|_{2 n}=\left\|d_{2 n} x^{2} d_{2 n}\right\|_{\mathcal{B}\left(\ell_{2}\right)}=\left\|d_{2 n} x\right\|_{\mathcal{B}\left(\ell_{2}\right)}^{2},
$$

and by [1, Proposition II.1.4.2],

$$
\|x\|_{n}=\left\|d_{n} x d_{n}\right\|_{\mathcal{B}\left(\ell_{2}\right)}=\nu\left(d_{n} x d_{n}\right)=\nu\left(d_{2 n} x\right) \leqslant\left\|d_{2 n} x\right\|_{\mathcal{B}\left(\ell_{2}\right)},
$$

where $\nu(\cdot)$ denotes the spectral radius. This gives the desired inequality.

(iii) Since $\mathcal{S} \hookrightarrow \mathcal{B}\left(\ell_{2}\right)$, any $x \in \mathcal{S}$ is also a Hilbert space operator, and the block-matrix operator $\left[\begin{array}{cc}\left(x x^{*}\right)^{1 / 2} & x \\ x^{*} & \left(x^{*} x\right)^{1 / 2}\end{array}\right]$ is positive in $\mathcal{B}\left(\ell_{2} \oplus \ell_{2}\right)$ (see e.g. [10, p. 117]). Equivalently,

$$
|\langle x \xi, \eta\rangle|^{2} \leqslant\left\langle\left(x x^{*}\right)^{1 / 2} \eta, \eta\right\rangle\left\langle\left(x^{*} x\right)^{1 / 2} \xi, \xi\right\rangle \quad \forall \xi, \eta \in \ell_{2} .
$$

For $m \in \mathbb{N}$, let us write $p_{m}:=\left[\begin{array}{cc}I_{m} & 0 \\ 0 & 0\end{array}\right]$ where $I_{m} \in \mathcal{M}_{m}$ is the identity matrix. Now fix $n \in \mathbb{N}$ and choose $\xi, \eta \in H_{n}^{\prime}$. Then $p_{m} \xi, p_{m} \eta \in \ell_{2}$ for all $m \in \mathbb{N}$ and (2) gives

$$
\left|\left\langle p_{m} x p_{m} \xi, \eta\right\rangle\right|^{2} \leqslant\left\langle p_{m}\left(x x^{*}\right)^{1 / 2} p_{m} \eta, \eta\right\rangle\left\langle p_{m}\left(x^{*} x\right)^{1 / 2} p_{m} \xi, \xi\right\rangle .
$$

Since $\left(p_{m}\right)_{m \in \mathbb{N}}$ is an approximate identity in $\mathcal{S}$ (see [13, Proposition 2]), we obtain

$$
|\langle x \xi, \eta\rangle|^{2} \leqslant\left\langle\left(x x^{*}\right)^{1 / 2} \eta, \eta\right\rangle\left\langle\left(x^{*} x\right)^{1 / 2} \xi, \xi\right\rangle .
$$

Taking the supremum over all $\xi, \eta$ in the unit ball of $H_{n}^{\prime}$ we get

$$
\|x\|_{n}^{2} \leqslant\left\|\left(x x^{*}\right)^{1 / 2}\right\|_{n}\left\|\left(x^{*} x\right)^{1 / 2}\right\|_{n} .
$$

Applying (ii) to the positive operators $\left(x x^{*}\right)^{1 / 2}$ and $\left(x^{*} x\right)^{1 / 2}$ we conclude that $\|x\|_{n}^{2} \leqslant\left\|x^{*} x\right\|_{2 n}^{\frac{1}{2}}\left\|x x^{*}\right\|_{2 n}^{\frac{1}{2}}$.

For sharpness, observe that if $x$ is a diagonal rank one matrix unit then we have equality in both (ii) and (iii). 
Proposition 3. For any $n, m \in \mathbb{N}$ and $x_{1}, \ldots, x_{m}, y_{1}, \ldots, y_{m} \in \mathcal{S}$, we have

$$
\begin{aligned}
& \sum_{j=1}^{m}\left\|x_{j}\right\|_{n}\left\|y_{j}\right\|_{n} \\
& \leqslant \frac{\pi^{2}}{6}\left\|\sum_{j=1}^{m} x_{j}^{*} x_{j}\right\|_{2 n+1}^{\frac{1}{4}}\left\|\sum_{j=1}^{m} x_{j} x_{j}^{*}\right\|_{2 n+1}^{\frac{1}{4}}\left\|\sum_{j=1}^{m} y_{j}^{*} y_{j}\right\|_{2 n+1}^{\frac{1}{4}}\left\|\sum_{j=1}^{m} y_{j} y_{j}^{*}\right\|_{2 n+1}^{\frac{1}{4}} .
\end{aligned}
$$

Proof. Let $C:=\frac{\pi^{2}}{6}$ and let $p \in \mathbb{N}$. We claim that

$$
\sum_{k=1}^{m}\left\|x_{k}^{*} x_{k}\right\|_{p} \leqslant C\left\|\sum_{k=1}^{m} x_{k}^{*} x_{k}\right\|_{p+1} .
$$

By the Cauchy-Schwarz inequality and Proposition 2(iii) this will then imply the desired inequality. To establish the claim, let $\xi^{1}, \ldots, \xi^{m} \in U_{p}^{\circ}$ and let us write $\left(e_{j}\right)_{j \in \mathbb{N}}$ for the standard basis vectors in $\ell^{2}$. We have

$$
\sum_{k=1}^{m}\left\langle x_{k}^{*} x_{k} \xi^{k}, \xi^{k}\right\rangle=\sum_{k=1}^{m} \sum_{i, j=1}^{+\infty}\left\langle x_{k}^{*} x_{k} e_{j}, e_{i}\right\rangle(i j)^{p} \overline{\xi_{i}^{k}} i^{-p} \xi_{j}^{k} j^{-p} .
$$

Applying the Cauchy-Schwarz inequality to summation over $i, j \in \mathbb{N}$ gives

$$
\sum_{k=1}^{m}\left\langle x_{k}^{*} x_{k} \xi^{k}, \xi^{k}\right\rangle \leqslant \sum_{k=1}^{m}\left(\sum_{i, j=1}^{+\infty}\left|\left\langle x_{k}^{*} x_{k} e_{j}, e_{i}\right\rangle\right|^{2}(i j)^{2 p}\right)^{\frac{1}{2}} .
$$

Since $x^{*} x$ is positive for any $x \in \mathcal{S}$, and for positive operators $y \in \mathcal{S}$ we have $\left|y_{i j}\right|^{2} \leqslant y_{i i} y_{j j}$ (where $y_{i j}:=\left\langle y e_{j}, e_{i}\right\rangle$ ), this implies that

$$
\begin{aligned}
\sum_{k=1}^{m}\left\langle x_{k}^{*} x_{k} \xi^{k}, \xi^{k}\right\rangle & \leqslant \sum_{j=1}^{+\infty}\left\langle\left(\sum_{k=1}^{m} x_{k}^{*} x_{k}\right) j^{p} e_{j}, j^{p} e_{j}\right\rangle \\
& \leqslant \sum_{j=1}^{+\infty} j^{-2} \sup _{i \in \mathbb{N}}\left\langle\left(\sum_{k=1}^{m} x_{k}^{*} x_{k}\right) i^{p+1} e_{i}, i^{p+1} e_{i}\right\rangle \\
& \leqslant C\left\|\sum_{k=1}^{m} x_{k}^{*} x_{k}\right\|_{p+1} .
\end{aligned}
$$

By Proposition 2(i), for any $\varepsilon>0$ there are $\xi^{1}, \ldots, \xi^{m} \in U_{p}^{\circ}$ with

$$
\sum_{k=1}^{m}\left\|x_{k}^{*} x_{k}\right\|_{p}<\sum_{k=1}^{m}\left\langle x_{k}^{*} x_{k} \xi^{k}, \xi^{k}\right\rangle+\varepsilon<C\left\|\sum_{k=1}^{m} x_{k}^{*} x_{k}\right\|_{p+1}+\varepsilon
$$

Taking the infimum over $\varepsilon>0$ yields the claim. 
As a straightforward consequence of Proposition 3, we obtain:

Theorem 4 (Grothendieck's inequality in $\mathcal{S}$ ). There is a constant $K \leqslant \frac{\pi^{2}}{6}$ such that $\|z\|_{\pi, n} \leqslant 2 K\|z\|_{a h, 2 n+1}$ for any $n \in \mathbb{N}$ and $z \in$ $\mathcal{S} \otimes \mathcal{S}$. Moreover, every continuous bilinear form $u: \mathcal{S} \times \mathcal{S} \rightarrow \mathbb{C}$ satisfies inequality (1) with $k=2 n+1$, for any $n, m \in \mathbb{N}$ and any $x_{1}, \ldots, x_{m}, y_{1}, \ldots, y_{m} \in \mathcal{S}$. In particular, taking $u(x, y):=\phi(x) \phi(y)$ where $\phi \in \mathcal{S}^{\prime}$, we obtain

$$
\sum_{j=1}^{m}\left|\phi\left(x_{j}\right)\right|^{2} \leqslant K\left(\|\phi\|_{n}^{*}\left\|\left(x_{j}\right)\right\|_{2 n+1}^{\mathrm{RC}}\right)^{2}
$$

Remark. This shows that $\kappa(n) \leqslant 2 n+1$. On the other hand, it is easy to show that $\kappa(n)>2 n-1$. Indeed, if not, then (3) would hold with $2 n+1$ replaced by some $\ell \leqslant 2 n-1$. Take $m \in \mathbb{N}$, define $\xi_{m}:=\sum_{j=1}^{m} j^{n} e_{j}$ and $\phi_{m} \in \mathcal{S}^{\prime}$ by $\phi_{m}(x):=\left\langle x \xi_{m}, \xi_{m}\right\rangle$. Then for $x_{j}:=$ $e_{j j}, j=1, \ldots, m$ we get $\left\|\left(x_{j}\right)\right\|_{\ell}^{\mathrm{RC}}=m^{\ell}$ and $\left\|\phi_{m}\right\|_{n}^{*}=m$. On the other hand, $\sum_{j=1}^{m}\left|\phi_{m}\left(x_{j}\right)\right|^{2}$ is equivalent (up to a constant) to $m^{4 n+1}$. Therefore (3) takes the form $m^{4 n+1} \leqslant C m^{2 \ell+2}$ for some constant $C$ (independent of $m$ ). Letting $m$ tend to infinity, we obtain $\ell \geqslant 2 n-\frac{1}{2}$, a contradiction. Hence $\kappa(n) \in\{2 n, 2 n+1\}$.

\section{Optimality}

We will now show that $\kappa(n)=2 n+1$. For this, we will use the tensor product formulation, noting that

$$
\kappa(n)=\min \left\{k \in \mathbb{N}: \sup \left\{\frac{\|z\|_{\pi, n}}{\|z\|_{a h, k}}: z \in \mathcal{S} \otimes \mathcal{S}, z \neq 0\right\}<\infty\right\} .
$$

Recall the diagonal operator $d_{n}$ defined on page 4 above. Since every $x \in \mathcal{S}$ is an operator on $\ell_{2}$ via the canonical inclusions $\ell_{2} \stackrel{d_{n}}{\hookrightarrow} H_{n}^{\prime} \stackrel{x}{\rightarrow}$

$H_{n} \stackrel{d_{n}}{\leftrightarrow} \ell_{2}$, it is clear that if $x \in \mathcal{S}$, then $d_{n} x$ and $x d_{n}$ are both operators on $\ell_{2}$. This leads to the following observation.

Proposition 5. If $z=\sum_{j=1}^{k} x_{j} \otimes y_{j} \in \mathcal{S} \otimes \mathcal{S}$, then

$$
\|z\|_{\pi, n}=\left\|\sum_{j=1}^{k} d_{n} x_{j} d_{n} \otimes d_{n} y_{j} d_{n}\right\|_{\pi} .
$$

Proof. Write

$$
\Delta_{n} z=\sum_{j=1}^{k} d_{n} x_{j} d_{n} \otimes d_{n} y_{j} d_{n} \in \mathcal{B}\left(\ell_{2}\right) \otimes \mathcal{B}\left(\ell_{2}\right) .
$$


If $\Delta_{n} z=\sum_{l=1}^{m} a_{l} \otimes b_{l} \in \mathcal{B}\left(\ell_{2}\right) \otimes \mathcal{B}\left(\ell_{2}\right)$ and $\sum_{l=1}^{m}\left\|a_{l}\right\|\left\|b_{l}\right\|<\left\|\Delta_{n} z\right\|_{\pi}+\varepsilon$ for some $\varepsilon>0$, then $z=\sum_{l=1}^{m} d_{n}^{-1} a_{l} d_{n}^{-1} \otimes d_{n}^{-1} b_{l} d_{n}^{-1}$ and

$$
\|z\|_{\pi, n} \leqslant \sum_{l=1}^{m}\left\|d_{n}^{-1} a_{l} d_{n}^{-1}\right\|_{n}\left\|d_{n}^{-1} b_{l} d_{n}^{-1}\right\|_{n}=\sum_{l=1}^{m}\left\|a_{l}\right\|\left\|b_{l}\right\|<\left\|\Delta_{n} z\right\|_{\pi}+\varepsilon .
$$

This gives $\|z\|_{\pi, n} \leqslant\left\|\Delta_{n} z\right\|_{\pi}$. The reverse inequality is proved similarly.

We also need the following well-known fact.

Proposition 6. If $\mathcal{H}$ is a Hilbert space and $x_{1}, \ldots, x_{m} \in \mathcal{B}(\mathcal{H})$, then

$$
\left\|\sum_{j=1}^{m} x_{j} \otimes x_{j}^{*}\right\|_{h}=\left\|\sum_{j=1}^{m} x_{j} x_{j}^{*}\right\| .
$$

Proof. By [15, Theorem 4.3], the Haagerup norm on the left hand side is equal to the completely bounded norm of the map on $\mathcal{B}(\mathcal{H})$ given by $a \mapsto \sum_{j=1}^{m} x_{j} a x_{j}^{*}$, which is completely positive, so attains its completely bounded norm at the identity operator.

Theorem 7. For every $n \in \mathbb{N}$, we have $\kappa(n)=2 n+1$.

Proof. By Theorem 4, it only remains to show that $\kappa(n)>2 n$. Choose $k_{n} \in \mathbb{N}$ sufficiently large that $k+1 \leqslant 2^{k}\left(k^{\frac{1}{4 n}}-1\right)$ for all $k \geqslant k_{n}$. This inequality ensures that for every $k \geqslant k_{n}$, if we define

$$
i_{1}=2^{k}, \quad i_{k+1}=\left\lfloor k^{\frac{1}{4 n}} 2^{k}\right\rfloor, \quad i_{j}=i_{k+1}+j-(k+1), 2 \leqslant j \leqslant k,
$$

then $i_{1}<i_{2}<\cdots<i_{k+1}$. Denote by $\left(e_{i j}\right)_{i, j \in \mathbb{N}}$ the standard matrix units, and for $j=2, \ldots, k+1$, consider the self-adjoint operators

$$
x_{j}:=e_{i_{1}, i_{j}}+e_{i_{j}, i_{1}} \in \mathcal{M}_{i_{k+1}} \subset \mathcal{S} \text {. }
$$

Let

$$
z_{k}:=\sum_{j=2}^{k+1} x_{j} \otimes x_{j}
$$

Since $d_{n} x_{j} d_{n}=i_{1}^{n} i_{j}^{n}\left(e_{i_{1}, i_{j}}+e_{i_{j}, i_{1}}\right)$ and $\left(d_{n} x_{j} d_{n}\right)^{2}=i_{1}^{2 n} i_{j}^{2 n}\left(e_{i_{1}, i_{1}}+e_{i_{j}, i_{j}}\right)$, by Propositions 5 and 6 we obtain

$$
\begin{aligned}
\left\|z_{k}\right\|_{\pi, n} & =\left\|\sum_{j=2}^{k+1} d_{n} x_{j} d_{n} \otimes d_{n} x_{j} d_{n}\right\|_{\pi} \\
& \geqslant\left\|\sum_{j=2}^{k+1} d_{n} x_{j} d_{n} \otimes d_{n} x_{j} d_{n}\right\|_{h}=\left\|\sum_{j=2}^{k+1}\left(d_{n} x_{j} d_{n}\right)^{2}\right\|=i_{1}^{2 n} \sum_{j=2}^{k+1} i_{j}^{2 n} .
\end{aligned}
$$


On the other hand,

$$
\left|x_{j}\right|^{2}=x_{j}^{2}=e_{i_{1}, i_{1}}+e_{i_{j}, i_{j}} \quad \text { and } \quad \sum_{j=2}^{k+1} d_{2 n} x_{j}^{2} d_{2 n}=i_{1}^{4 n} k e_{i_{1}, i_{1}}+\sum_{j=2}^{k+1} i_{j}^{4 n} e_{i_{j}, i_{j}} .
$$

Therefore

$$
\begin{aligned}
\left\|z_{k}\right\|_{a h, 2 n} & \leqslant\left\|\sum_{j=2}^{k+1}\left|x_{j}\right|^{2}\right\|_{2 n}=\left\|\sum_{j=2}^{k+1} d_{2 n} x_{j}^{2} d_{2 n}\right\|=\max \left\{i_{1}^{4 n} k, i_{k+1}^{4 n}\right\} \\
& \leqslant i_{1}^{4 n} k+i_{k+1}^{4 n} .
\end{aligned}
$$

Hence

$$
\frac{\left\|z_{k}\right\|_{\pi, n}}{\left\|z_{k}\right\|_{a h, 2 n}}>\frac{i_{1}^{2 n} \sum_{j=2}^{k+1} i_{j}^{2 n}}{i_{1}^{4 n} k+i_{k+1}^{4 n}}>\frac{i_{1}^{-2 n} i_{2}^{2 n}}{1+k^{-1} i_{1}^{-4 n} i_{k+1}^{4 n}} \rightarrow \infty \text { as } k \rightarrow \infty,
$$

by our choice of $i_{1}, \ldots, i_{k+1}$. So $\kappa(n)>2 n$ as required.

\section{Reformulations of The INEQUALity}

Here we give several different ways of stating our inequality; in each case, an analogous result may be found in [12]. The methods here are fairly standard, so full proofs are often omitted. Throughout, we write $K=\sup _{n \in \mathbb{N}} K_{n} \leqslant \pi^{2} / 6$.

4.1. Grothendieck's inequality with states. Given $\xi \in U_{n}^{\circ}$, let $\phi_{\xi} \in \mathcal{S}^{\prime}$ be given by $\phi_{\xi}(x)=\langle x \xi, \xi\rangle, x \in \mathcal{S}$. We call an element of the closed convex hull of $\left\{\phi_{\xi}: \xi \in U_{n}^{\circ}\right\}$ an n-state on $\mathcal{S}$. Note that by Proposition 2(i), for any positive element $x \in \mathcal{S}$ we have $\|x\|_{n}=$ $\sup \left\{\phi(x): \phi \in V_{n}\right\}$, where $V_{n} \subseteq \mathcal{S}^{\prime}$ is the set of all $n$-states on $\mathcal{S}$. The next result may be deduced from Theorem 4 by closely following the Hahn-Banach Separation argument of $[12, \S 23]$.

Theorem 8. For any continuous bilinear form $u: \mathcal{S} \times \mathcal{S} \rightarrow \mathbb{C}$ and $n \in \mathbb{N}$, there are $(2 n+1)$-states $\phi_{1}, \phi_{2}, \psi_{1}, \psi_{2}$ on $\mathcal{S}$ with

$$
|u(x, y)| \leqslant K\|u\|_{n}^{*}\left(\phi_{1}\left(x^{*} x\right)+\phi_{2}\left(x x^{*}\right)\right)^{\frac{1}{2}}\left(\psi_{1}\left(y^{*} y\right)+\psi_{2}\left(y y^{*}\right)\right)^{\frac{1}{2}}
$$

for all $x, y \in \mathcal{S}$.

4.2. 'Little' Grothendieck inequality. As a consequence we obtain the following 'little' Grothendieck inequality in $\mathcal{S}$. Recall that if $T: X \rightarrow Y$ is a linear map between Fréchet spaces, then $\|T\|_{n, k}:=$ $\sup \left\{\|T x\|_{k}:\|x\|_{n} \leqslant 1\right\}$. 
Theorem 9. For any Fréchet-Hilbert space $H$, if $u_{1}, u_{2}: \mathcal{S} \rightarrow H$ are continuous linear maps, $k, m, n \in \mathbb{N}$ and $x_{1}, \ldots, x_{m}, y_{1}, \ldots, y_{m} \in \mathcal{S}$, then

$$
\left|\sum_{j=1}^{m}\left\langle u_{1}\left(x_{j}\right), u_{2}\left(y_{j}\right)\right\rangle_{k}\right| \leqslant K\left\|u_{1}\right\|_{n, k}\left\|u_{2}\right\|_{n, k}\left\|\left(x_{j}\right)\right\|_{2 n+1}^{\mathrm{RC}}\left\|\left(y_{j}\right)\right\|_{2 n+1}^{\mathrm{RC}} .
$$

Equivalently, for any $k, n \in \mathbb{N}$ there are $(2 n+1)$-states $\phi_{1}, \phi_{2}, \psi_{1}, \psi_{2}$ such that for all $x, y \in \mathcal{S}$ we have

$$
\begin{aligned}
\left|\left\langle u_{1}(x), u_{2}(y)\right\rangle_{k}\right| \leqslant K & \left\|u_{1}\right\|_{n, k}\left\|u_{2}\right\|_{n, k} \\
& \times\left(\phi_{1}\left(x^{*} x\right)+\phi_{2}\left(x x^{*}\right)\right)^{\frac{1}{2}}\left(\psi_{1}\left(y^{*} y\right)+\psi_{2}\left(y y^{*}\right)\right)^{\frac{1}{2}} .
\end{aligned}
$$

Proof. Apply Theorems 4 and 8 to $u_{k}(x, y):=\left\langle u_{1}(x), u_{2}(y)\right\rangle_{k}$ for $k \in$ $\mathbb{N}$.

Using the same argument as in the proof of Theorem 8 we can obtain an equivalent version of the 'little' Grothendieck inequality.

Theorem 10. For any Fréchet-Hilbert space $H$, if $u: \mathcal{S} \rightarrow H$ is a continuous linear map and $k, n \in \mathbb{N}$, then there exist $(2 n+1)$-states $\phi_{1}, \phi_{2}$ on $\mathcal{S}$ such that for all $x \in \mathcal{S}$ we have

$$
\|u x\|_{k} \leqslant \sqrt{K}\|u\|_{n, k}\left(\phi_{1}\left(x^{*} x\right)+\phi_{2}\left(x x^{*}\right)\right)^{\frac{1}{2}} .
$$

\section{REFERENCES}

[1] B. Blackadar. Operator algebras, volume 122 of Encyclopaedia of Mathematical Sciences. Springer-Verlag, Berlin, 2006.

[2] T. Ciaś. On the algebra of smooth operators. Studia Math., 218(2):145-166, 2013.

[3] P. Domański. Algebra of smooth operators. http://main3. amu.edu.pl/ domanski/salgebra1.pdf, 2012.

[4] M. Fragoulopoulou. Topological algebras with involution. Elsevier Science B.V., Amsterdam, 2005.

[5] A. Grothendieck. Résumé de la théorie métrique des produits tensoriels topologiques. Resenhas, 2(4):401-480, 1996. Reprint of Bol. Soc. Mat. São Paulo 8 (1953), 1-79.

[6] U. Haagerup. The Grothendieck inequality for bilinear forms on $C^{*}$-algebras. Adv. in Math., 56(2):93-116, 1985.

[7] H. Jarchow. Locally convex spaces. B. G. Teubner, 1981. 
[8] S. Kaijser and A.M. Sinclair. Projective tensor products of $C^{*}$ algebras. Math. Scand., 55(2):161-187, 1984.

[9] R. Meise and D. Vogt. Introduction to functional analysis, volume 2 of Oxford Graduate Texts in Mathematics. Oxford University Press, 1997.

[10] V. Paulsen. Completely bounded maps and operator algebras. Cambridge University Press, Cambridge, 2002.

[11] G. Pisier. Introduction to operator space theory, volume 294 of London Mathematical Society Lecture Note Series. Cambridge University Press, Cambridge, 2003.

[12] G. Pisier. Grothendieck's theorem, past and present. Bull. Amer. Math. Soc. (N.S.), 49(2):237-323, 2012.

[13] K. Piszczek. Automatic continuity and amenability in the noncommutative Schwartz space. J. Math. Anal. Appl., 432(2):954964, 2015.

[14] K. Piszczek. A Jordan-like decomposition in the noncommutative Schwartz space. Bull. Aust. Math. Soc., 91(2):322-330, 2015.

[15] R. R. Smith. Completely bounded module maps and the Haagerup tensor product. J. Funct. Anal., 102(1):156-175, 1991.

School of Mathematics and Statistics, University College Dublin, Belfield, Dublin 4, Ireland

E-mail address: rupert.levene@ucd.ie

Faculty of Mathematics and Comp. Sci., A. Mickiewicz University in Poznań, Umultowska 87, 61-614 Poznań, Poland

E-mail address: kpk@amu.edu.pl 